\title{
The Status of the KSS Bound and its Possible Violations (How Perfect Can a Fluid Be?)
}

\author{
Antonio Dobado, Felipe J. Llanes-Estrada and Juan Miguel Torres Rincón \\ Depto. Física Teórica I, Universidad Complutense de Madrid \\ 28040 Madrid, Spain
}

\begin{abstract}
In this work we briefly review the Kovtun-Son-Starinet (KSS) computation of the ratio $\eta / s$ for quantum field theories with gravitational dual and the related conjecture that it is bound from below by $1 / 4 \pi$. We discuss the validity of the bound and the nature of its possible violations, its relevance for RHIC, its connection with phase transitions and other related issues.
\end{abstract}

Keywords: Viscosity over Entropy Density, KSS Holographic Conjecture, Heavy Ion Collisions.

PACS: 11.15.Pg, 12.38.Mh, 25.75.q, 51.20.+d

\section{INTRODUCTION}

Probably one of the most astonishing and relevant discoveries of the last decade in theoretical physics was the AdS/CFT (Anti de Sitter/Conformal Field Theory) correspondence [1]. This powerful tool makes it possible to get deep insights into the dynamics of strongly interacting gauge theories with a gravity dual in the limit $N_{c} \rightarrow \infty$ and $\lambda \rightarrow \infty$, where $N_{c}$ is the number of colors and $\lambda$ is the 't Hooft coupling $g^{2} N_{c}$. According to this holographic correspondence it is possible to relate some black-brane configurations in higher dimensions to certain four dimensional conformal Quantum Field Theories (CFT) at finite temperature. The thermodynamic properties such as temperature and entropy of the CFT can thus be obtained from the corresponding Bekenstein definitions for the black-brane. Moreover, not only the thermodynamics but also the hydrodynamic behavior of the black-brane horizon can be identified with the CFT hydrodynamics. In particular, by using the AdS/CFT correspondence it has been possible to compute, for a very large class of four dimensional theories with gravity duals, the ratio [2]

$$
\frac{\eta}{s}=\frac{1}{4 \pi}
$$

where $\eta$ is the shear viscosity and $s$ is the entropy density, dimensionally both scaling as $\left[E^{3}\right]$ in natural units. From the CFT point of view, the standard relativistic hydrodynamic equations [3], $\partial_{\mu} T^{\mu v}=0$ for $T^{\mu v}$, the energy momentum tensor, can be understood as the effective theory describing the dynamics of the CFT at large distance and time [4]. Following the philosophy of the effective theories it is possible to expand the energymomentum tensor in powers of the space-time derivatives. To zeroth order we get the well known ideal fluid equations. Dissipative processes require going to the next order where diffusion coefficients such as the shear viscosity $\eta$, the bulk viscosity $\zeta$ and the heat conductivity $\kappa$ appear together with other possible diffusion coefficients $D_{i}$. 
From the linearized equation of motion in momentum space at this order one can get the dispersion relation of the mechanical excitations, which are two transverse modes with

$$
\omega(k)=-i \frac{\eta}{\varepsilon+P} k^{2},
$$

(where $\varepsilon$ is the energy density and $P$ the pressure) and one sound mode

$$
\omega(k)=c_{s}-\frac{i}{2}\left(\frac{4}{3} \eta+\zeta\right) \frac{k^{2}}{\varepsilon+P},
$$

where $c_{s}=\sqrt{d P / d \varepsilon}$ is the speed of sound. The imaginary part in the right hand-side entails a damping of the corresponding mode, and its size therefore measures the ability of the fluid to dissipate perturbations. Since for vanishing chemical potential we have the thermodynamic equation $\varepsilon+P=T s, \eta / s$ governs the right hand side and is a good way to characterize the intrinsic ability of a system to relax towards equilibrium [5], especially for $\zeta=0$ which is, in particular, the case of CFT.

\section{COMPUTING $\eta / s$ FROM THE ADS/CFT CORRESPONDENCE}

How is it possible to compute the diffusion coefficients? The traditional way is by using kinetic theory, more precisely the Boltzmann equation or its quantum version, the Uehling-Uhlenbenck equation, written in terms of the elastic cross-sections, and which can be solved by the Chapman-Enskog method to first order in the perturbation out of equilibrium [6]. When can we then apply these kinetic theory methods?

The main condition is that the mean free path must be much larger than the interaction distance, which typically occurs for low density, weakly interacting, systems. Then one can find the well known and somewhat counter-intuitive result establishing that the greater the interaction, the lesser the viscosity.

From a more modern prospective, it is possible to use the so called Kubo formulae. The corresponding shear viscosity (for short, viscosity in the following) can be obtained by analyzing linear response theory and coupling the fluid to gravity by using an appropriate lightly curved space-time background. Then one can find

$$
\eta=\lim _{\omega \rightarrow 0}\left(\frac{1}{2 \omega} \int d t d \bar{x} e^{i \omega t}\left\langle\left[T_{x y}(t, \bar{x}), T_{x y}(0, \overline{0})\right]\right\rangle\right) .
$$

This equation can be used to compute $\eta / s$ in the context of the AdS/CFT correspondence. In order to do that one starts from a CFT with gravity dual. For example for $\mathscr{N}=4, S U\left(N_{c}\right)$ Super Yang-Mills one can consider the metric in five-dimensional AdS

$$
d s^{2}=\frac{r^{2}}{R^{2}}\left[-\left(1-\frac{r_{0}^{4}}{r^{4}}\right) d t^{2}+d x^{2}+d y^{2}+d z^{2}\right]+\frac{R^{2}}{r^{2}\left(1-r_{0}^{4} / r^{4}\right)} d r^{2} .
$$

The dual theory is a CFT at temperature $T$ equal to the Hawking temperature of the black-brane and Bekenstein entropy $S=A / 4 G_{N}$, where $A$ is the (hyper)area of the blackbrane horizon located at $r=r_{0}$. Now, following Klebanov [7], consider a graviton polarized in the $\mathrm{x}-\mathrm{y}$ direction and propagating perpendicularly to the brane. The absorption 
cross-section of the graviton by the brane measures, in the dual CFT, the imaginary part of the retarded Green's function of the operator coupled to the metric i.e. the energymomentum tensor. Then it is possible to find

$$
\eta=\frac{\sigma(0)}{16 \pi G_{N}}
$$

where $\sigma(0)$ is the graviton absorption cross-section at zero energy. This cross-section can be computed classically by using linerized Einstein equations and it turns out equal to the horizon area so that one finally arrives to the formula shown in Eq.(1). Quite remarkable is that the result then does not depend on the particular form of the metric. It is the same for Dp, M2 and M5 branes. Basically the reason is the universality of the graviton absorption cross-section.

\section{THE CONJECTURE OF KOVTUN, SON AND STARINETS}

From the result for the $\eta / s$ ratio for theories with a gravity dual, Kovtun, Son and Starinets (KSS) proposed the conjecture that, for a very wide class of systems, including those that can be described by a sensible (i.e. ultraviolet complete) quantum field theory, the above ratio has the lower bound

$$
\frac{\eta}{s} \geq \frac{1}{4 \pi}
$$

There are several pieces of evidence supporting this conjecture. The first one is based on the Heisenbeg uncertainty principle and kinetic theory. The viscosity is proportional to the energy density $\varepsilon$ and the mean free time $\tau$. On the other hand the entropy density is proportional to the number density $n$. Therefore $\eta / s \sim \varepsilon \tau / n \sim E \tau$ where $E$ is the average particle energy. Thus, from the Heisenberg principle for time-energy, we obtain the bound modulo the numerical constant.

Another important hint to establish the bound comes from the computation done by Buchel, Liu and Starinets [8] where they obtained the leading correction to the $\eta / s$ parameter in inverse powers of the 't Hooft coupling using the $\alpha^{\prime}$ corrected low energy effective action for the type IIB string theory, dual to the $\mathscr{N}=4, S U\left(N_{c}\right)$ SYM. They found the beautiful result

$$
\frac{\eta}{s}=\frac{1}{4 \pi}\left[1+\frac{135 \zeta(3)}{8\left(2 g^{2} N_{c}\right)^{3 / 2}}+\ldots\right],
$$

where $\zeta(z)$ is the Riemann $\zeta$-function so that $\zeta(3)=1.2020569 \ldots$ is the Apéry constant. Therefore the correction to the ratio $\eta / s$ is positive and diverges for the 't Hooft coupling $\lambda$ approaching zero, and saturates the bound for $\lambda$ growing to infinity. Assuming a smooth extrapolation between the two regimes one can infer the validity of the bound for any value of $\lambda$. Finally, we have knowledge of no fluid that undercomes the bound. For all fluids examined so far, the $\eta / s$ ratio is well above the bound for the range of measured temperatures and pressures. This includes also superfluid helium and even trapped ${ }^{6} \mathrm{Li}$ at strong coupling [9]. 
An obvious consequence of the KSS conjecture (if correct), is the absence of perfect fluids in Nature, since the entropy density will only vanish at absolute zero. Is this physically acceptable? Of course, ideal fluids have been a source of interesting paradoxes.

Already in non relativistic fluid dynamics one encounters the d'Alembert paradox (an ideal fluid with no boundaries exerts no force on a body moving through it, i.e. there is no lift force). From it follows the unconformable impossibility of flying or swimming. Of course, the difficulty disappears if there are no perfect fluids.

As a more recent conceptual difficulty of ideal fluids, we recall that it has been pointed out that the accretion of an ideal fluid onto a black hole could violate the Generalized Second Law of Thermodynamics [10], suggesting a possible connection between this law and the KSS bound.

In conclusion, the existence of a minimum viscosity would put to rest a number of problems in fluid mechanics, so the bound is not unwelcome by theorists.

\section{THE RHIC CASE}

The $3834 \mathrm{~m}$ long Relativistic Heavy Ion Collider (RHIC) is operated at the Brookhaven National Laboratory (BNL) for, among others, Au+Au collisions $(A=197)$, and can reach a center of mass energy per nucleon of $E=200 \mathrm{GeV}$ with a luminosity $\mathscr{L}=$ $2 \times 10^{26} \mathrm{~cm}^{-2} \mathrm{~s}^{-1}$. It has four experiments called STAR, PHENIX, BRAHMS and PHOBOS. We highlight some (preliminary) results from RHIC:

- Thermochemical models describe well the different particle yields fitting to $T=$ $177 \mathrm{MeV}$ and baryon chemical potential $\mu_{B}=29 \mathrm{MeV}$ for $E_{C M}=200 \mathrm{GeV}$ per nucleon.

- From observed transversity and rapidity distributions, the Bjorken model predicts an energy density at time $t_{0}=1 \mathrm{fm}$ of $4 \mathrm{GeV} \mathrm{fm}^{-3}$ whereas the critical density is about $0.7 \mathrm{GeV} \mathrm{fm}^{-3}$, i.e. the matter created may be well above the threshold for Quark Gluon Plasma (QGP) formation.

- A surprising amount of collective flow is observed in the outgoing hadrons [11], both in the single particle transverse momentum distribution (radial flow) and in the asymmetric azimuthal distribution around the beam axis (elliptic flow).

For our purposes, the main conclusions derivable from these preliminary results are:

- Fluid dynamics with very low viscosity reproduces the measurements of radial and elliptic flow up to transverse momenta of $1.5 \mathrm{GeV}$.

- Collective flow is probably generated early in the collision, probably in the QGP phase before hadronization. The QGP seems to be more strongly interacting than expected on the basis of perturbative QCD and asymptotic freedom (hence the low viscosity). An alternative exists, fast, instability-driven, equilibration [12].

- Some preliminary estimations of $\eta / s$ based on elliptic flow $[11,13]$ and transverse momentum correlations [14] seem to be compatible with value close to 0.08 (the KSS bound). This would make it the most perfect fluid known. 


\section{CAN THE KSS BOUND BE VIOLATED?}

Several avenues to theoretically break the KSS bound have been pursued. If one tries to undercome a bound on $\eta / s$, the possible strategies are to either decrease the viscosity at fixed entropy density, or to increase the entropy density at fixed viscosity. We now briefly comment on these attempts and their status.

\section{Increase the Entropy Mixing Several Species}

This possibility was realized already in [15] and has been exploited in detail in [16] not without some controversy [17]. The basic idea is that in principle it could be possible to avoid the KSS bound in a non-relativistic system with constant cross section and a large number $g$ of non-identical, degenerate particles, by increasing the Gibbs mixing entropy.

It is possible that the KSS bound applies only to systems that can be obtained from a "sensible" (UV complete) QFT. Is it possible to find a non-relativistic system coming from a sensible QFT that violates the KSS bound for large degeneracy? As an interesting example we can consider the case of a massive Non Linear Sigma Model (NLSM) based on the coset $S O(g+1) / S O(g)$. Then in the non relativistic limit it is possible to find [18]

$$
\frac{\eta}{s}=\frac{80 \sqrt{2} \pi^{3}}{11} \frac{f^{4}}{m^{4}} \frac{m}{T} \frac{1}{n \lambda^{3}\left(\log \frac{g}{n \lambda^{3}}+\frac{5}{2}\right)},
$$

where $m$ is the mass of the $g$ degenerated pseudo Goldstone bosons, $f$ is the NLSM scale parameter, $n$ is the particle number density and $\lambda$ is the thermal de Broglie wavelength $\lambda=\sqrt{2 \pi / m T}$. The above result is valid only in the region $T \ll m, n \lambda^{3} \ll g$ and $m \sim f$. Inside this region the KSS bound is satisfied provided $g$ is not very large, but it fails if $g$ is exponentially large.

The NLSM is not an UV complete theory but in principle we have at least two ways to complete it.

First, simply QCD, since the above NLSM is the lagrangian of Chiral Perturbation Theory at the lowest order with $g=3$ for $N_{f}=2$ degenerated quarks since $S U(2) \times$ $S U(2) / S U(2)=S O(4) / S O(3)$. This corresponds to having three Goldstone bosons (pions). Then it would appear that increasing the number of the QCD flavors, which implies increasing the number of Goldstone bosons, one could undermine the KSS bound according to the formula above. However this is not the case since, as it is well known, one cannot increase the QCD flavor number without changing the sign of the derivative of the $\beta$ function at the origin, $\beta(g)=-g^{3}\left(11-2 N_{f} / 3\right) / 16 \pi^{2}+$.. (here $g$ is the QCD coupling, not the pion degeneracy) and presumably QCD is not well defined outside the asymptotic freedom regime. In addition the above formula applies only for NLSM coset $S O(g+1) / S O(g)$ whilst the chiral QCD coset is $S U\left(N_{f}\right)_{L} \times$ $S U\left(N_{f}\right)_{R} / S U\left(N_{f}\right)_{L+R}$. Both families of cosets meet only for $g=3$ and $N_{f}=2$ but not in the general case. Thus the above results do not describe low-energy QCD for $g$ different from 3 . 
The other possibility for completing the NLSM is by introducing the corresponding Linear Sigma Model (LSM) since the NLSM can be considered as the low energy limit of the LSM for large $\sigma$ (Higgs) masses and the LSM is, at least perturbatively a well defined renormalizable QFT. In this case, and for the same regime of validity than in the NLSM, we get the same result for $\eta / s$. Thus, for an exponentially large $g$ we can violate the KSS bound. Is there any way out for the KSS conjecture? In principle there is one. The above computation only considered binary interactions, but even at very small temperatures we have an exponential number of non-elastic processes which make the system metastable with a very weak tendency to relax to a state where the density is fixed by the condition $\mu=m$ and where the result above does not apply any more. Another possibility to keep the validity of the KSS bound is the almost established triviality of the LSM which would ruin this way to complete the NLSM through a sensible QFT theory.

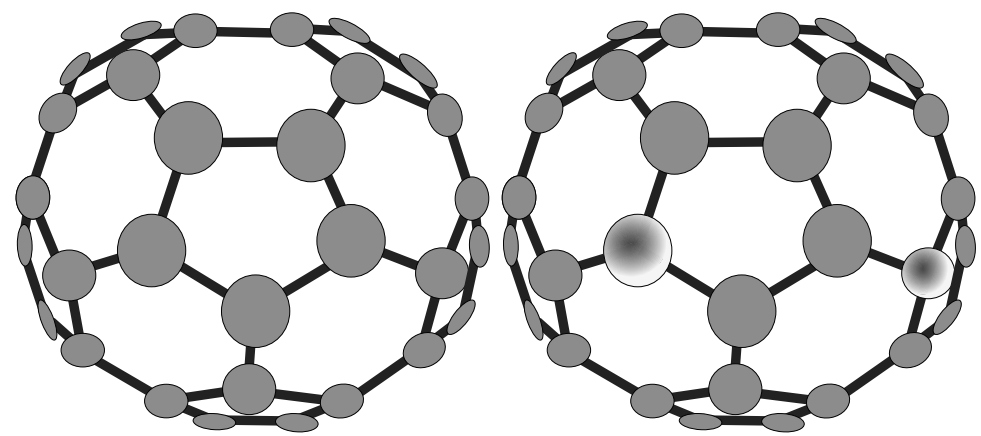

FIGURE 1. A way to violate the KSS bound is by employing the logarithmic increase of the mixing entropy in a multicomponent gas. This might be achieved with a factorially large number of molecules, here a cartoon of fullerene with two substitutions. However, simple estimates [18] indicate that it will be difficult to achieve fast enough growth of the number of species with the molecule size (since the sublimation temperature and hence viscosity also grow with the size).

The concept of multiple substitutions in complex molecules, yielding an exponentially (factorially) large number of isomers, is illustrated in figure 1. Although we have not been able to clearly identify a family of currently synthesyzed molecules, because of the complicated interplay of tightness of binding, excluded volume, sublimation temperature, etc. that vary together with the number of substitutable nodes, the possibility remains a priori. It is not clear how the argument of ultraviolet completion and a discussion about the completion of the standard model would affect a non-relativistic gas (the relevant case for most of our physical reality). From a practical point of view, although we know of no fluid that violates the bound, it is not obvious how this empirical fact is tied to the "weak" form of the conjecture that requires good UV behavior, since by arguments of scale separation, the low-energy effective theory would be all needed to describe reality to a given precision. Investigations continue. 


\section{Decrease the Viscosity by Altering the Dual Gravitational Action}

Another possible source of violation of the bound comes from considering changes of the scattering cross-sections in the gas that decrease the viscosity.

From the point of view of the AdS/CFT correspondence, the bound is saturated for standard general relativity. However, on general grounds, one expects to have higher derivative corrections to the Einstein-Hilbert action. For example one could consider the case of a theory with a gravity dual described at low energy by a Gauss-Bonnet gravity action

$$
S_{G B}=\frac{1}{16 \pi G_{N}}=\int d^{5} x \sqrt{-g}\left[R-2 \Lambda+\frac{\lambda_{G B}}{2} L^{2}\left(R^{2}-4 R_{\mu v} R^{\mu v}+R_{\mu v \rho \sigma} R^{\mu v \rho \sigma}\right)\right],
$$

where $\Lambda=-6 / L^{2}$. In this case it is possible to find ${ }^{1}[19]$

$$
\frac{\eta}{s}=\frac{1}{4 \pi}\left(1-4 \lambda_{G B}\right) \text {. }
$$

This result is non perturbative in $\lambda_{G B}$ and shows that the bound can be violated for positive $\lambda_{G B}$. One could argue that this result is suspicious since it does not make sense for $\lambda_{G B}>4$. However in [20] it is shown that, in order to avoid microcausality violations in the corresponding CFT we need to have

$$
\frac{\eta}{s} \geq \frac{16}{25}\left(\frac{1}{4 \pi}\right)
$$

This leaves room for violation of the KSS bound but requires $\lambda_{G B} \leq 9 / 100$ for the Gauss-Bonnet theory to be consistent. However there could be other additional consistency constraints in the theory forcing the $\lambda_{G B}$ parameter to vanish or be negative thus reestablishing the validity of the KSS bound.

\section{Unitarity Invalidates Certain Reported Violations}

From the QFT point of view, a way to decrease the viscosity without affecting the entropy in excess is to increase the interparticle interaction. This has been remarked in the past, in particular in the context of the pion gas. We however have shown that the method fails because unitarity imposes a bound on the cross-section.

Thus, the extrapolation of the low-energy effective theory for pions (ChPT to first or second order) to higher energies, ceases to be valid when resonances in the meson gas are reached $(\sigma, \rho$, etc.). A properly unitarized method such as the Inverse Amplitude Method (IAM) has been reported in reference [21] and is plotted in figure 2, where we have extended our past calculations to include strange mesons $(K, \eta)$ and their lowest resonances through the $S U(3)$ IAM [22].

\footnotetext{
${ }^{1}$ The authors thank Juan Maldacena for bringing our attention to these references.
} 

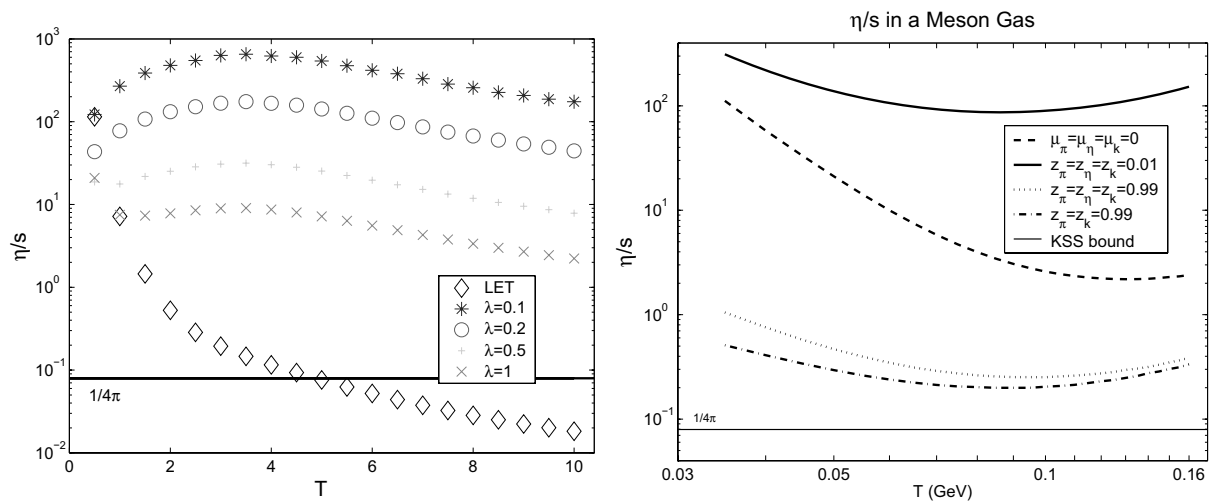

FIGURE 2. Reported violations of the $\eta / s$ holographic bound in the meson gas at high temperature are spurious: in this graph we show how the cross section computed within unitarized Chiral Perturbation Theory by means of the Inverse Amplitude Method, respects the bound $1 / 4 \pi$ for all conceivable temperatures that the hadron gas might attain (right). Violations reported are due to an unphysically small (large) viscosity (cross section) induced by unitarity violations. For comparison, we show a similar calculation with the unitarized Linear Sigma Model (left) that yields the same result.

\section{$\eta / s$ AND THE PHASE TRANSITION}

Recently, Csernai, Kapusta and McLerran [23] made the observation that, in all systems whose $\frac{\eta}{s}(T)$ plot has been examined, the minimum of $\eta / s$ and the liquid-gas phase transition happen at the same temperature. This fact can be roughly understood because in a gas, as the temperature increases, there is more efficient momentum transport and then the viscosity goes to infinity. However in a liquid, which can be seen as a mixture of clusters and voids, molecules swap voids less efficiently at low temperature. Then as temperature decreases there are less voids and consequently more shear momentum transport due to adhesivity among layers, so that the viscosity takes also off to infinity.

Therefore somewhere between the two phases the viscosity should have a minimum. Empirically, for standard fluids, $\eta / s$ is found to reach its minimum at or near the critical temperature. Thus, apparently there is a connection between $\eta / s$ and the phase transition but we do not have any clear theory of this [24]. For example we ignore if there are universal critical exponents for the different kind of phase transitions. A very interesting possibility is that the same behavior observed in ordinary liquids could also occur in QCD. If this were the case, an experimental determination of the $\eta / s$ minimum could give information about the QCD phase transition and whether there is a critical endpoint. This direction has been explored in [25].

The hadron-gas phase has been examined with great care by us [24] and close colleagues [26, 27], so that great improvements are not to be expected. The quark and gluon plasma phase, however, is less known in the strong-coupling regime and requires more work. The situation is represented in figure 3. At high baryon chemical potential one would expect a jump in the ratio $\eta / s$ (by analogy with common substances) at a first order phase transition, but a continuous minimum at low baryon-density where one 


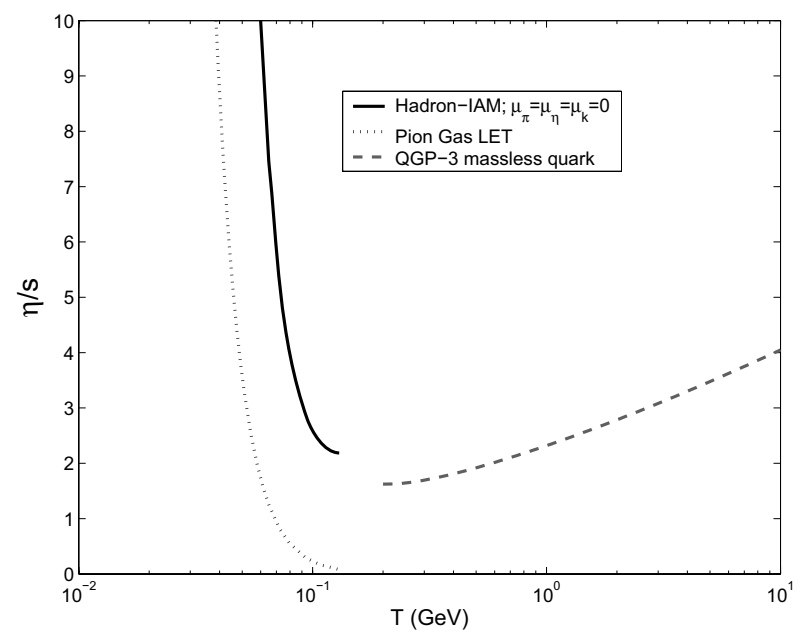

FIGURE 3. By analogy with common, human-scale fluids, $\eta / s$ is expected to have a minimum but not a discontinuity at the predicted cross-over between the hadron phase and the quark and gluon plasma phase. We have reexamined the computation in the hadron phase with more detailed than past work, but a discontinuity remains, maybe due to the uncertainties in the quark-gluon plasma side. This observable is a promising tag of a possible critical end point in the QCD phase-diagram.

expects a cross-over. Current computations of $\eta / s$ from the hadron phase are quite mature, but the quark-gluon plasma has not yet been refined beyond high- $T$ perturbation theory.

\section{CONCLUSIONS AND OPEN QUESTIONS}

The AdS/CFT correspondence makes possible to study new aspects of QFT such as viscosity and other hydrodynamic behavior. The KSS bound set a new limit on how perfect a fluid can be coming from holography which was completely unexpected. From the experimental stand point there is no counter example for this bound. From the RHIC data we observe a large amount of collective flow that can be properly described by hydrodynamic models with low viscosity compatible with the KSS bound. Some theoretical models suggest that unitarity could be related in some way with the KSS bound. There is a theoretical counter example of the bound in a non-relativistic model with large degeneracy. However possibly the model is not UV complete because of the triviality of the LSM. This could be an indication that a more precise formulation of the bound is needed.

Some open questions are the following: Is the bound correct for some well defined formulation? Could it be possible to really measure $\eta / s$ at RHIC with precision enough to check the KSS bound? Are there any connections between the KSS and the entropy Bekenstein bounds? How are the minima of $\eta / s$ related to phase transitions? Could it be considered an order parameter? 


\section{ACKNOWLEDGMENTS}

This work has been partially supported by the DGICYT (Spain) under grants FPA 200402602 and FPA 2005-02327 and by the Universidad Complutense/CAM, project number 910309 and BSCH-PR34/07-15875. A. D. thanks José Edelstein and the organization for their kind invitation to participate in this celebration of the tenth anniversary of the discovery of the AdS/CFT correspondence held at such a great place as Buenos Aires, and Alex Buchel and Juan Maldacena for useful comments.

\section{REFERENCES}

1. J. M. Maldacena, "The large $\mathrm{N}$ limit of superconformal field theories and supergravity," $A d v$. Theor. Math. Phys. 2, 231 (1998) [Int. J. Theor. Phys. 38, 1113 (1999)] [arXiv:hep-th/9711200];

S. S. Gubser, I. R. Klebanov and A. M. Polyakov, "Gauge theory correlators from non-critical string theory," Phys. Lett. B 428, 105 (1998) [arXiv:hep-th/9802109];

E. Witten, "Anti-de Sitter space and holography," Adv. Theor. Math. Phys. 2, 253 (1998) [arXiv:hepth/9802150];

E. Witten, "Anti-de Sitter space, thermal phase transition, and confinement in gauge theories," $A d v$. Theor. Math. Phys. 2, 505 (1998) [arXiv:hep-th/9803131].

2. G. Policastro, D. T. Son and A. O. Starinets, "The shear viscosity of strongly coupled $\mathrm{N}=4$ supersymmetric Yang-Mills plasma," Phys. Rev. Lett. 87, 081601 (2001) [arXiv:hep-th/0104066];

P. Kovtun, D. T. Son and A. O. Starinets, "Holography and hydrodynamics: Diffusion on stretched horizons," JHEP 0310, 064 (2003) [arXiv:hep-th/0309213].

3. L.D. Landau and E. M. Lifshitz, Fluid Mechanics, Pergamon Press, Oxford, 1987.

4. D. T. Son and A. O. Starinets, "Viscosity, Black Holes, and Quantum Field Theory," Ann. Rev. Nucl. Part. Sci. 57, 95 (2007) [arXiv:0704.0240 [hep-th]].

5. P. Danielewicz and M. Gyulassy, "Dissipative Phenomena In Quark Gluon Plasmas," Phys. Rev. D 31, 53 (1985).

6. A. Dobado and F. J. Llanes-Estrada, "The viscosity of meson matter," Phys. Rev. D 69, 116004 (2004) [arXiv:hep-ph/0309324].

7. I. R. Klebanov, "World-volume approach to absorption by non-dilatonic branes," Nucl. Phys. B 496, 231 (1997) [arXiv:hep-th/9702076].

8. A. Buchel, J. T. Liu and A. O. Starinets, "Coupling constant dependence of the shear viscosity in N=4 supersymmetric Yang-Mills theory," Nucl. Phys. B 707, 56 (2005) [arXiv:hep-th/0406264].

9. T. Schafer, "The Shear Viscosity to Entropy Density Ratio of Trapped Fermions in the Unitarity Limit," Phys. Rev. A 76, 063618 (2007) [arXiv:cond-mat/0701251];

G. Rupak and T. Schafer, "Shear viscosity of a superfluid Fermi gas in the unitarity limit," arXiv:0707.1520 [cond-mat.other].

10. I. Fouxon, G. Betschart and J. D. Bekenstein, Phys. Rev. D 77, 024016 (2008) [arXiv:0710.1429 [gr-qc]].

11. E. Shuryak, "Why does the quark gluon plasma at RHIC behave as a nearly ideal fluid?," Prog. Part. Nucl. Phys. 53, 273 (2004) [arXiv:hep-ph/0312227].

12. S. Mrowczynski, "Scenario Of Instabilities Driven Equilibration Of The Quark-Gluon Plasma," Eur. Phys. J. A 31, 875 (2007).

13. D. Teaney, "Effect of shear viscosity on spectra, elliptic flow, and Hanbury Brown-Twiss radii," Phys. Rev. C 68, 034913 (2003) [arXiv:nucl-th/0301099].

14. S. Gavin and M. Abdel-Aziz, "Measuring Shear Viscosity Using Transverse Momentum Correlations in Relativistic Nuclear Collisions," Phys. Rev. Lett. 97, 162302 (2006) [arXiv:nucl-th/0606061].

15. P. Kovtun, D. T. Son and A. O. Starinets, "Viscosity in strongly interacting quantum field theories from black hole physics," Phys. Rev. Lett. 94, 111601 (2005) [arXiv:hep-th/0405231].

16. T. D. Cohen, "Is there a 'most perfect fluid' consistent with quantum field theory?," Phys. Rev. Lett. 99, 021602 (2007) [arXiv:hep-th/0702136];

A. Cherman, T. D. Cohen and P. M. Hohler, "A sticky business: the status of the cojectured viscos- 
ity/entropy density bound," JHEP 0802, 026 (2008) [arXiv:0708.4201 [hep-th]].

17. D. T. Son, "Comment on 'Is There a 'Most Perfect Fluid' Consistent with Quantum Field Theory?'," Phys. Rev. Lett. 100, 029101 (2008) [arXiv:0709.4651 [hep-th]].

18. A. Dobado and F. J. Llanes-Estrada, "On the violation of the holographic viscosity versus entropy KSS bound in non relativistic systems," Eur. Phys. J. C 51, 913 (2007) [arXiv:hep-th/0703132].

19. Y. Kats and P. Petrov, "Effect of curvature squared corrections in AdS on the viscosity of the dual gauge theory," arXiv:0712.0743 [hep-th];

M. Brigante, H. Liu, R. C. Myers, S. Shenker and S. Yaida, "Viscosity Bound Violation in Higher Derivative Gravity," arXiv:0712.0805 [hep-th].

20. M. Brigante, H. Liu, R. C. Myers, S. Shenker and S. Yaida, "The Viscosity Bound and Causality Violation," arXiv:0802.3318 [hep-th].

21. A. Dobado and F. J. Llanes-Estrada, "The ratio of viscosity to entropy density in a pion gas satisfies the KSS holographic bound," Eur. Phys. J. C 49, 1011 (2007) [arXiv:hep-ph/0609255].

22. J. R. Pelaez and A. Gomez Nicola, "Unitarization of the complete meson meson scattering at one loop in chiral perturbation theory," AIP Conf. Proc. 602, 34 (2001) [arXiv:hep-ph/0109074].

23. L. P. Csernai, J. I. Kapusta and L. D. McLerran, "On the strongly-interacting low-viscosity matter created in relativistic nuclear collisions," Phys. Rev. Lett. 97, 152303 (2006) [arXiv:nucl-th/0604032].

24. A. Dobado, F. J. Llanes-Estrada and J. M. Torres-Rincon, " $\eta / s$ and the phase transition of the NonLinear Sigma Model," arXiv:0803.3275 [hep-ph].

25. R. A. Lacey, N. N. Ajitanand, J. M. Alexander, P. Chung, J. Jia, A. Taranenko and P. Danielewicz, "An estimate for the location of QCD critical end point," arXiv:0708.3512 [nucl-ex].

26. D. Fernandez-Fraile and A. G. Nicola, "Transport coefficients in chiral perturbation theory," Eur. Phys. J. A 31, 848 (2007) [arXiv:hep-ph/0610197].

27. D. Fernandez-Fraile and A. G. Nicola, "Transport properties of a meson gas," Int. J. Mod. Phys. E 16, 3010 (2007) [arXiv:0706.3561 [hep-ph]]. 
Copyright of AIP Conference Proceedings is the property of American Institute of Physics and its content may not be copied or emailed to multiple sites or posted to a listserv without the copyright holder's express written permission. However, users may print, download, or email articles for individual use. 